Supporting Information for:

\title{
Organic-to-Aqueous Phase Transfer of Cadmium Chalcogenide Quantum Dots using a Sulfur-Free Ligand for Enhanced Photoluminescence and Oxidative Stability
}

Raul Calzada ${ }^{\dagger}$, Christopher M. Thompson ${ }^{\dagger}$, Dana E. Westmoreland, Kedy Edme, and Emily A. Weiss*

$\dagger$ : Authors contributed equally to the work.

Department of Chemistry, Northwestern University, 2145 Sheridan Rd., Evanston, IL 602083113

*corresponding author. Email: e-weiss@northwestern.edu

Materials. All chemicals and solvents were purchased from Sigma-Aldrich and used asreceived.

Synthesis and Purification of Oleate-Capped CdS QDs. We prepared oleate-capped CdS QDs using a procedure modified from that of Harris et al. ${ }^{1}$ We added cadmium oxide $(0.36 \mathrm{~g}$, $2.80 \mathrm{mmol}$ ) to a solution of $90 \%$ technical grade oleic acid ( $4.4 \mathrm{~mL}, 13.9 \mathrm{mmol})$ in $90 \%$ technical grade octadecene $(14.0 \mathrm{~mL}, 43.8 \mathrm{mmol})$, and heated the mixture to $260^{\circ} \mathrm{C}$ under a $\mathrm{N}_{2}$ atmosphere with vigorous stirring until the solution became clear. Once the solution was clear, we injected elemental sulfur $(0.064 \mathrm{~g}, 2 \mathrm{mmol})$ as a solution in $4 \mathrm{~mL}$ of octadecene and allowed the nanoparticles to grow for 3 minutes at $250{ }^{\circ} \mathrm{C}$. To purify the QDs we added 3:1 (v:v) acetone to QD solution and centrifuged the mixture at $3500 \mathrm{rpm}$ for five minutes. We discarded the supernatant of octadecene and acetone and resuspended the pellet of QDs in hexanes. We then added 3:1 (v:v) methanol to the QD dispersion, centrifuged at $3500 \mathrm{rpm}$ for five minutes, discarded the supernatant, and resuspended the QDs in hexanes. The acetone and methanol washes were repeated one more time each before finally resuspending and storing the QDs in hexanes.

Synthesis and Purification of Oleate-Capped CdSe QDs. As in the synthesis of oleatecapped CdS QDs, we first synthesized cadmium oleate by adding cadmium oxide $(0.225 \mathrm{~g}, 1.75$ $\mathrm{mmol}), 90 \%$ technical grade oleic acid $(6.3 \mathrm{~mL}, 22.3 \mathrm{mmol})$, and $90 \%$ technical grade octadecene $(13.7 \mathrm{~mL}, 42.8 \mathrm{mmol})$ to a dry, three-neck round-bottom flask and then heated the mixture to 260 ${ }^{\circ} \mathrm{C}$ under an $\mathrm{N}_{2}$ atmosphere while stirring until the mixture turned clear. To synthesize the CdSe QDs we added $\mathrm{SeO}_{2}(0.09 \mathrm{~g}, 0.81 \mathrm{mmol})$ to $8 \mathrm{~mL}$ of cadmium oleate and $12 \mathrm{~mL}$ of $90 \%$ technical 
grade octadecene and heated the mixture to $240{ }^{\circ} \mathrm{C}$ under a $\mathrm{N}_{2}$ atmosphere. CdSe QDs began to form at $220^{\circ} \mathrm{C}$ and we allowed the reaction temperature to increase slowly until the CdSe QDs were the desired size. Longer times resulted in larger particles. We purified the QDs with an identical procedure as used for oleate-capped CdS QDs. This synthetic procedure was adapted from Chen et $_{\text {al. }}{ }^{2}$

Quantitative ${ }^{1} \mathbf{H}$ NMR. We performed all of our NMR experiments on an Agilent DD2 600 $\mathrm{MHz}$ spectrometer. We used the signal of hexamethylcyclotrisiloxane at $0.16 \mathrm{ppm}$, corresponding to its 18 equivalent protons, as an internal standard for experiments both in DMF-d7 and benzened6. For a $20 \mu \mathrm{M}$ solution of QDs in $0.75 \mathrm{~mL}$ of deuterated solvent, we used $11 \mu \mathrm{L}$ of a $30 \mu \mathrm{M}$ solution of hexamethylcyclotrisiloxane for a final concentration of $440 \mathrm{nM}$. Table S1 lists the measured quantities of free and bound oleate and PPA in various samples during the exchange.

We also quantified the amount of DMF left after the exchange into water by taking a spectrum of the PPA capped QDs in $\mathrm{D}_{2} \mathrm{O}$ with fumaric acid as internal standard (Figure S2). As described in the main text, we diluted $2 \mathrm{~mL}$ of PPA-capped QDs with $4 \mathrm{~mL}$ of $\mathrm{D}_{2} \mathrm{O}$ and extracted the DMF with 3 washes of $40 \mathrm{~mL}$ of $\mathrm{CHCl}_{3}$. We further diluted the QDs with 4 additional $\mathrm{mL}$ of $\mathrm{D}_{2} \mathrm{O}$ for a final volume of $8 \mathrm{~mL}$. We then prepared a $1 \mathrm{~mL}$ solution of QDs with $13.9 \mathrm{mM}$ fumaric acid. Integration of the fumaric acid vinyl peak at $6.5 \mathrm{ppm}(2 \mathrm{H}$; highlighted in purple) relative to the peaks of DMF at $7.92 \mathrm{ppm}, 2.99 \mathrm{ppm}$ and $2.84 \mathrm{ppm}(1 \mathrm{H}, 3 \mathrm{H}, 3 \mathrm{H}$; highlighted in green $)$ resulted in a final value of $4.2 \mathrm{mM}$ DMF left after the washing step.

Table S1. Quantification of ligands bound to QD surface and free in solution after exchange with PPA. ${ }^{a}$

\begin{tabular}{lccccc}
\hline & $\begin{array}{c}\text { Bound } \\
\text { Oleate } \\
(\mathrm{mmol})\end{array}$ & $\begin{array}{c}\text { Free oleate } \\
(\mathrm{mmol})\end{array}$ & $\begin{array}{c}\text { Free PPA } \\
(\mathrm{mmol})\end{array}$ & $\begin{array}{c}\Delta \mathrm{PPA} \\
(\mathrm{mmol})\end{array}$ & $\begin{array}{c}\text { QDs } \\
(\mathrm{mmol})^{b}\end{array}$ \\
\hline $\begin{array}{l}\text { QDs in C6 } 6 \text { 6 } \\
\text { pre- exchange }\end{array}$ & $4.69 \times 10^{-3}$ & $0.14 \times 10^{-3}$ & - & - & $1.55 \times 10^{-5}$ \\
$\begin{array}{l}\mathrm{C}_{6} \mathrm{D}_{6} \text { supernate } \\
\text { after exchange }\end{array}$ & - & $4.45 \times 10^{-3}$ & - & - & - \\
$\begin{array}{l}\text { QDs in DMF-d7 } \\
\text { after exchange }\end{array}$ & $2.97 \times 10^{-5}$ & $0.17 \times 10^{-3}$ & $1.42 \times 10^{-3}$ & $4.78 \times 10^{-3}$ & $1.28 \times 10^{-5}$ \\
\hline
\end{tabular}

${ }^{a}$ Number of ligands found by integration of ${ }^{1} \mathrm{H}$ NMR signals shown in Figure S1, relative to the signal of hexamethylcyclotrisiloxane as internal standard. 
${ }^{b}$ Number of QDs obtained from the absorbance of the peak of the first exciton, and the calibration curve from Yu et al. ${ }^{3}$

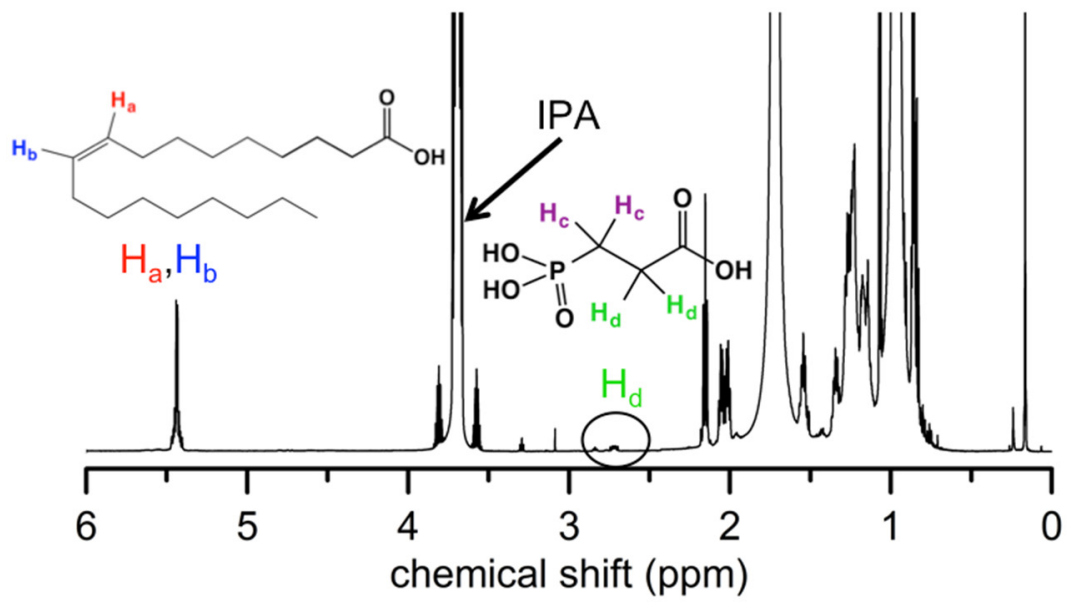

Figure S1. ${ }^{1} \mathrm{H}$ NMR spectrum of the supernatant of the exchange (step 2) in benzene-d6. The vinyl protons of oleate correspond to a sharp multiplet at $5.43 \mathrm{ppm}\left(\mathrm{H}_{\mathrm{a}, \mathrm{b}}\right)$. A small amount of free PPA is also present, and results in a signal at $2.7 \mathrm{ppm}\left(\mathrm{H}_{\mathrm{d}}\right)$. The signal for the $\mathrm{H}_{\mathrm{c}}$ protons in PPA is buried underneath the aliphatic signal of oleate. The sharp singlet at $0.16 \mathrm{ppm}$ corresponds to the 18 equivalent protons of hexamethylcyclotrisiloxane.

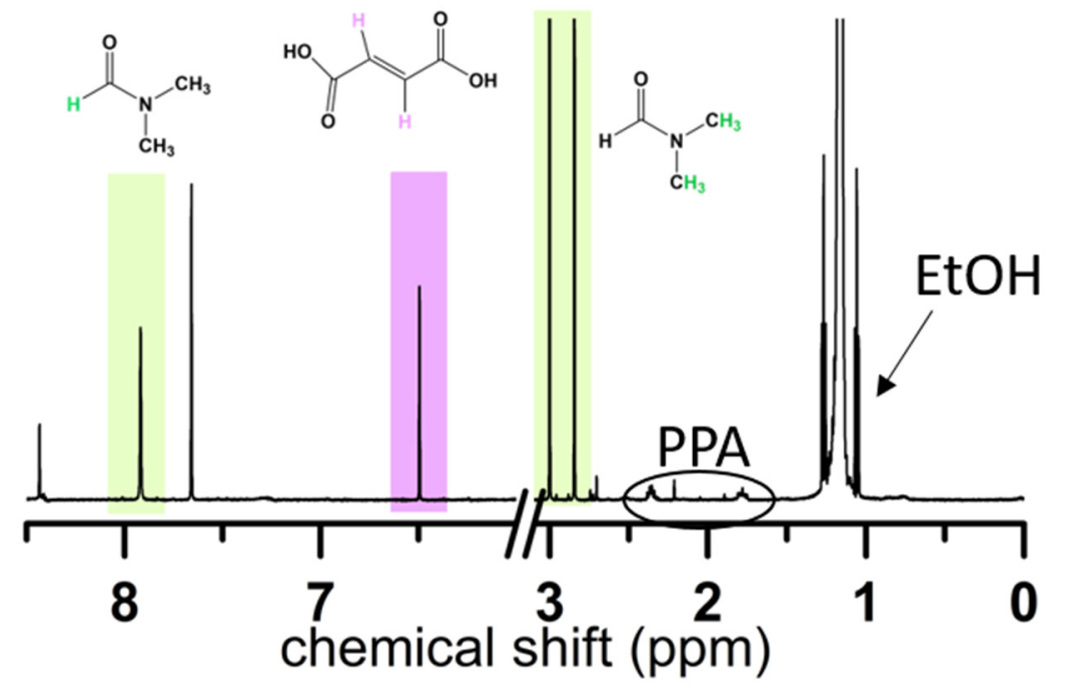

Figure S2. ${ }^{1} \mathrm{H}$ NMR spectrum of PPA-capped QDs in $\mathrm{D}_{2} \mathrm{O}$ after exchange through the procedure described in the main text. Fumaric acid (pictured with purple " $\mathrm{H}$ ") was used as an internal standard to quantify amount of DMF (pictured with green " $H$ ") left after extraction with 3 washes of $\mathrm{CHCl}_{3}$. A final concentration of $4.2 \mathrm{mM} \mathrm{DMF}$ in water was determined. Ethanol in the solution comes from the $\mathrm{CHCl}_{3}$, where it is used as stabilizer. 
Photoluminescence Quantum Yield Measurements. The relative photoluminescence (PL) quantum yield (QY) of CdS QDs transferred to water using our protocol was measured using anthracene dissolved in ethanol $(\mathrm{QY}=0.27)$ as a standard. The PL quantum yield of CdSe QDs was determined using Rhodamine $\mathrm{B}$ in ethanol $(\mathrm{QY}=0.65)$ as a standard. In all $\mathrm{QD}$ and anthracene/Rhodamine B samples, we diluted the sample such that the absorbance at the excitation wavelength ( $350 \mathrm{~nm}$ for $\mathrm{CdS}$ and $500 \mathrm{~nm}$ for CdSe) was less than 0.1 A.U. We used the tabulated fluorescence yield of anthracene in ethanol as a multiplicative factor (0.814) to correct for quenching of anthracene fluorescence by oxygen, as reported by Melhuish. ${ }^{4}$ The fluorescence yield of the QDs in water $\left(\Theta_{Q D}\right)$ was calculated using eq S1, where $\Theta_{r e f}$ is the PL QY of a fluorescent

$$
\Theta_{Q D}=\left(\frac{1-10^{-A_{a}}}{1-10^{-A_{Q D}}}\right) \times \frac{I_{Q D}}{I_{a}} \times \frac{\eta}{\eta_{r e f}} \times \Theta_{r e f}
$$

standard, $A_{a}$ and $A_{Q D}$ are the absorbance of the standard and QD sample at the excitation wavelength, $I_{a}$ is the integrated intensity of the fluorescence spectrum between $355-500 \mathrm{~nm}$ for anthracene and 520-700 $\mathrm{nm}$ for Rhodamine B, and $I_{Q D}$ is the integrated intensity of the QD bandedge PL between $408-470 \mathrm{~nm}$ for CdS and $524-615 \mathrm{~nm}$ for CdSe. $\eta$ and $\eta_{\text {ref }}$ are the refractive indices of water (1.333) and ethanol (1.359).

We observe, after correcting the spectra for lamp fluctuations, an S:N ratio in the PL spectrum of $>1000: 1$ for the most emissive samples. For the least emissive samples, for instance, MPAcoated CdS QDs in water, the S:N ratio is $\sim 2: 1$. Even with this S:N level, the PL peak is readily integrable such that the PL intensity is probably accurate within a factor of 2, whereas the differences between the QYs of various samples are often orders of magnitude. 

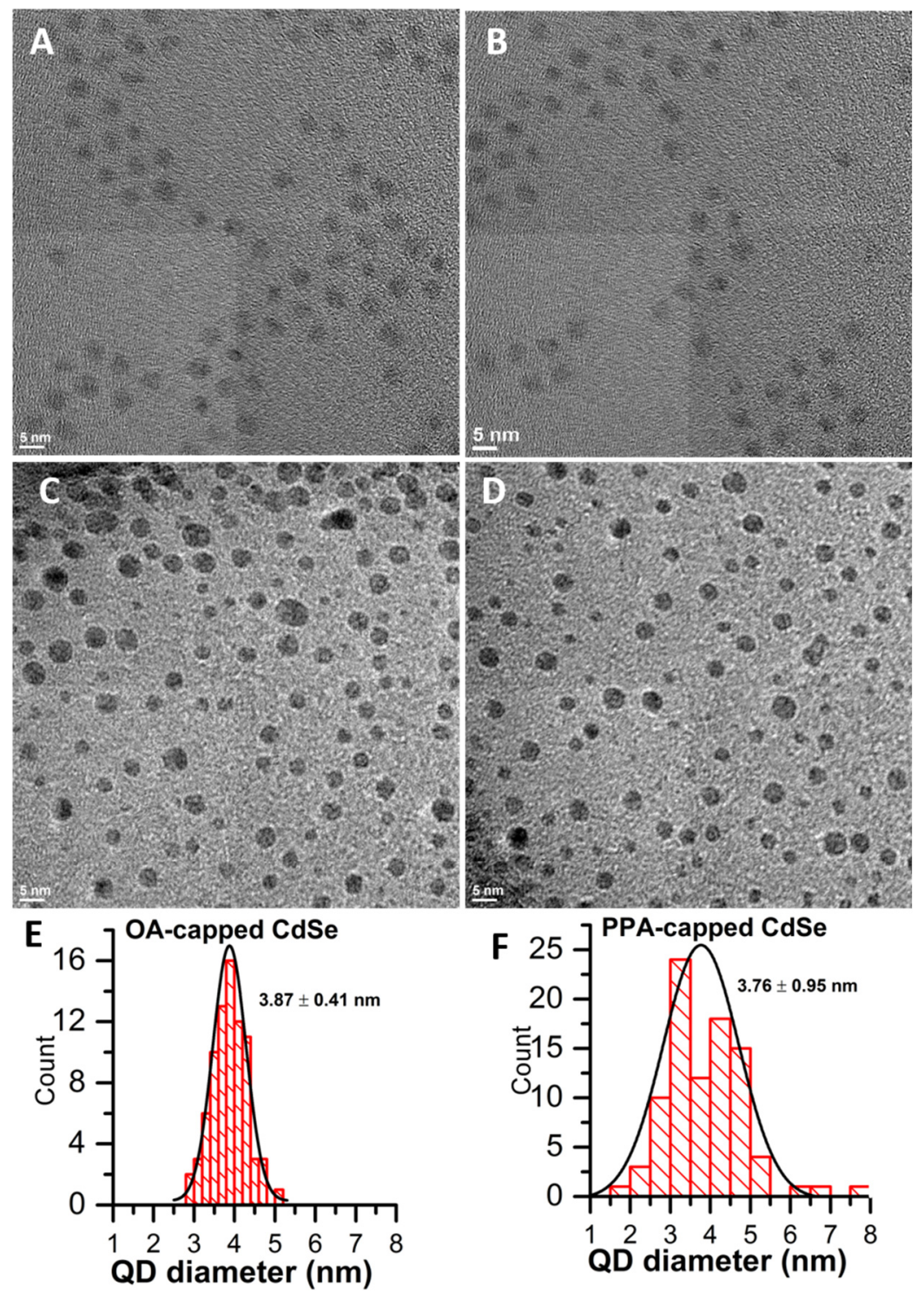

Figure S3. Transmission electron micrographs of oleate-capped CdSe QDs before phase transfer to water (A-B) and PPA-capped QDs after phase transfer (C-D). The bottom panel contains size histograms of oleate-capped CdSe (E, 80 particles) and PPA-capped CdSe (F, 90 particles) QDs. The average QD size and distribution were obtained from the Gaussian fits, pictured in the histograms.

Electrochemical Measurements. All cyclic voltammagrams (CVs) were recorded with a Princeton applied research potentiostat using a 3-mm radius glassy carbon working electrode, a platinum wire counter electrode, and a silver wire reference electrode. We used acetonitrile as our solvent for all samples and $0.08 \mathrm{M} \mathrm{NBu}_{4} \mathrm{PF}_{6}$ as supporting electrolyte. To calibrate our $\mathrm{CV}_{\mathrm{s}}$ to the 
SCE scale we also recorded the $\mathrm{CVs}$ with added $1 \mathrm{mM}$ ferrocene $\left(\mathrm{EFc}_{\mathrm{Fc}+}=+0.342 \mathrm{~V}\right.$ vs $\left.\mathrm{SCE}\right)$ as internal standard.

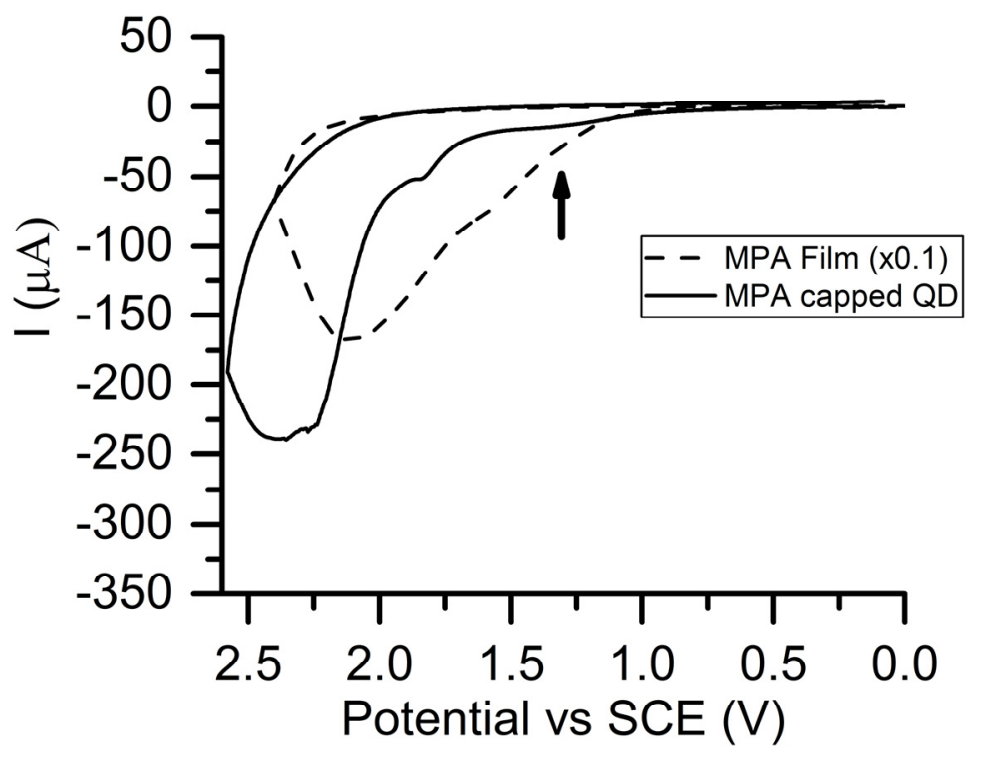

Figure S4. CV of films of MPA-capped QDs and MPA (free thiol) on glassy carbon electrode in acetonitrile. The scan rate was $0.1 \mathrm{~V} / \mathrm{s}$ scan rate an $0.08 \mathrm{M} \mathrm{NBu}_{4} \mathrm{PF}_{6}$ was the supporting electrolyte. The small peak in the MPA capped QDs at $+1.2 \mathrm{~V}$ vs SCE aligns with the onset potential of the film of the unbound thiol, and is therefore assigned to the oxidation of free ligand deposited along with the QDs.

Synthesis of Cd-MPA and Cd-PPA Complexes. We prepared Cd-MPA and Cd-PPA complexes by adding $\mathrm{CdCl}_{2}(18.3 \mathrm{mg}, 0.1 \mathrm{mmol})$ to a $5 \mathrm{~mL}, 20 \mathrm{mM}$, aqueous solution of PPA or MPA in a 1:1 ratio at $\mathrm{pH} 5$ and 11 respectively, to ensure that the binding group of the ligand was deprotonated. A white precipitate formed in both cases, which we centrifuged and washed with ethanol. Similar preparation methods for other Cd-phosphonate ${ }^{5}$ and Cd-thiolate ${ }^{6}$ complexes are reported in literature. 
Stability of PPA capped CdS and CdSe QDs as Measured by Absorption, PL, and CV.
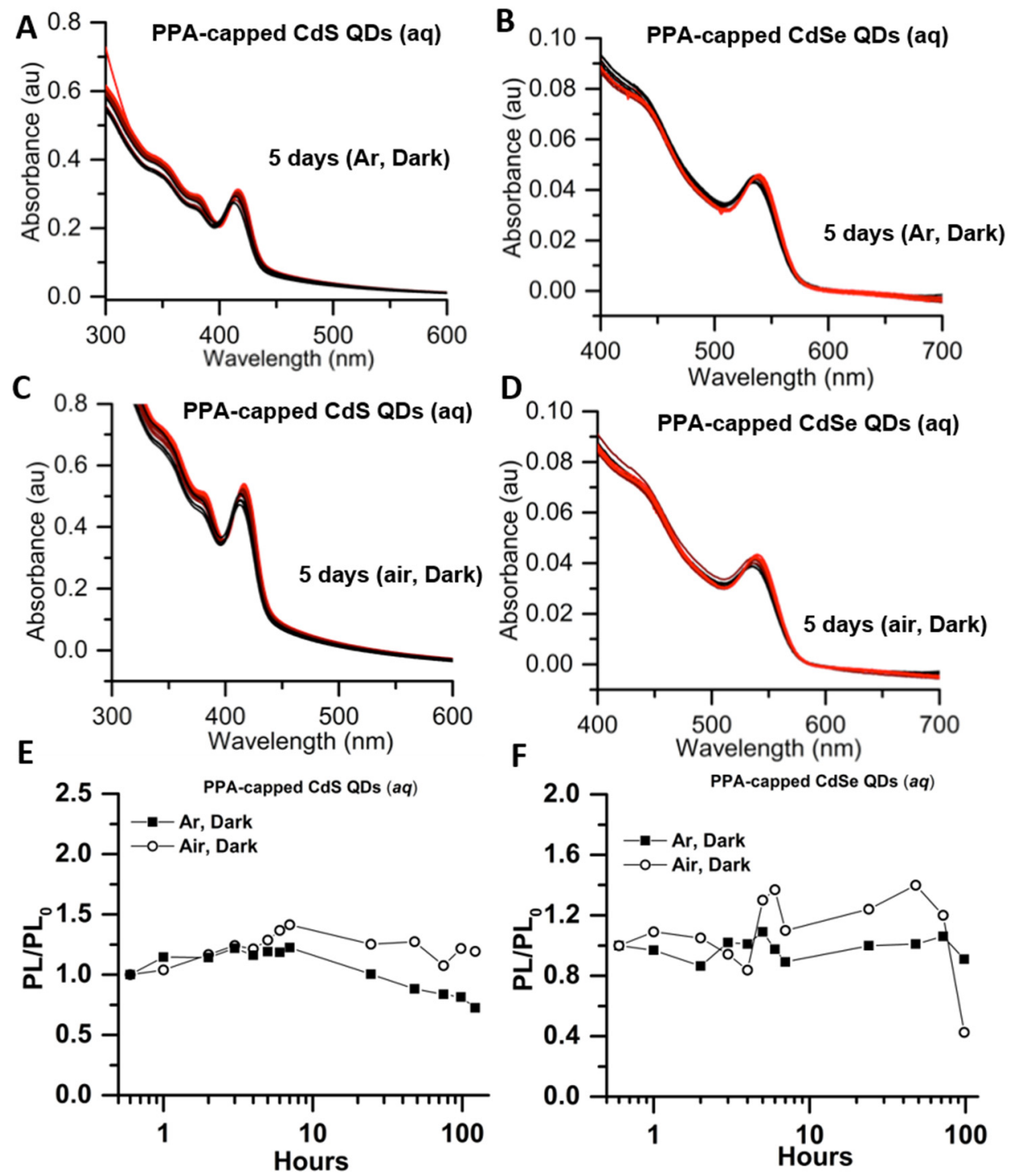

Figure S5. Absorbance over 5 days of PPA-capped CdS $(\mathbf{A}, \mathbf{C})$ and $\mathrm{CdSe}(\mathbf{B}, \mathbf{D}) \mathrm{QDs}$ in the dark under Ar, or in the dark in air. (E, F) PL intensity of the QD samples over 5 days, divided by their initial PL intensity (at time $0, \mathrm{PL}_{0}$ ), for $\mathrm{CdS}(\mathbf{E})$ and CdSe (F) QDs stored in the dark, under either air or Ar. 

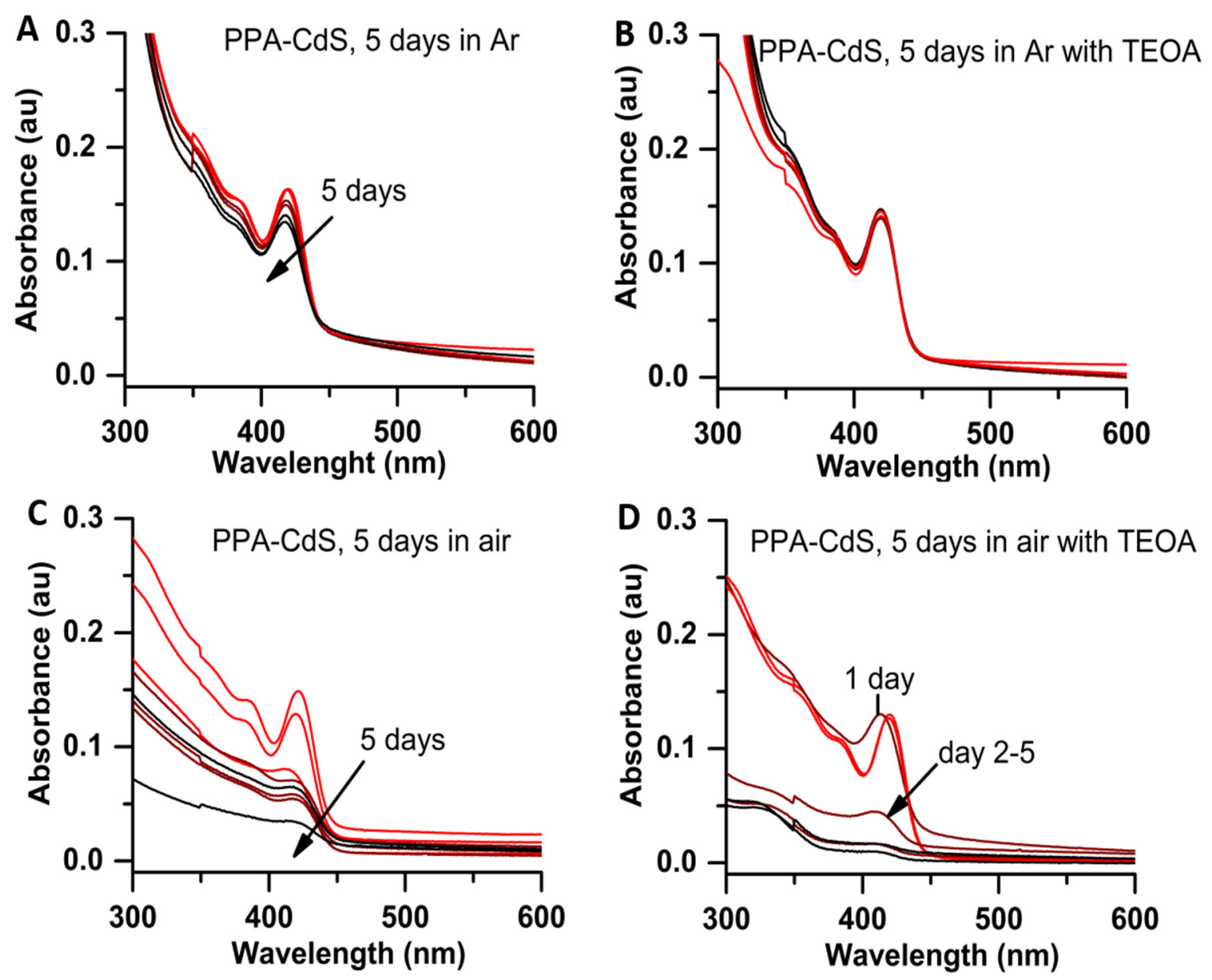

Figure S6. Absorbance of PPA-capped CdS QDs over 5 days stored in the light in $\operatorname{Ar}(\mathbf{A})$ and air $(\mathbf{C})$, and under the same conditions with TEOA as hole scavenger (B, Ar; D, air). 

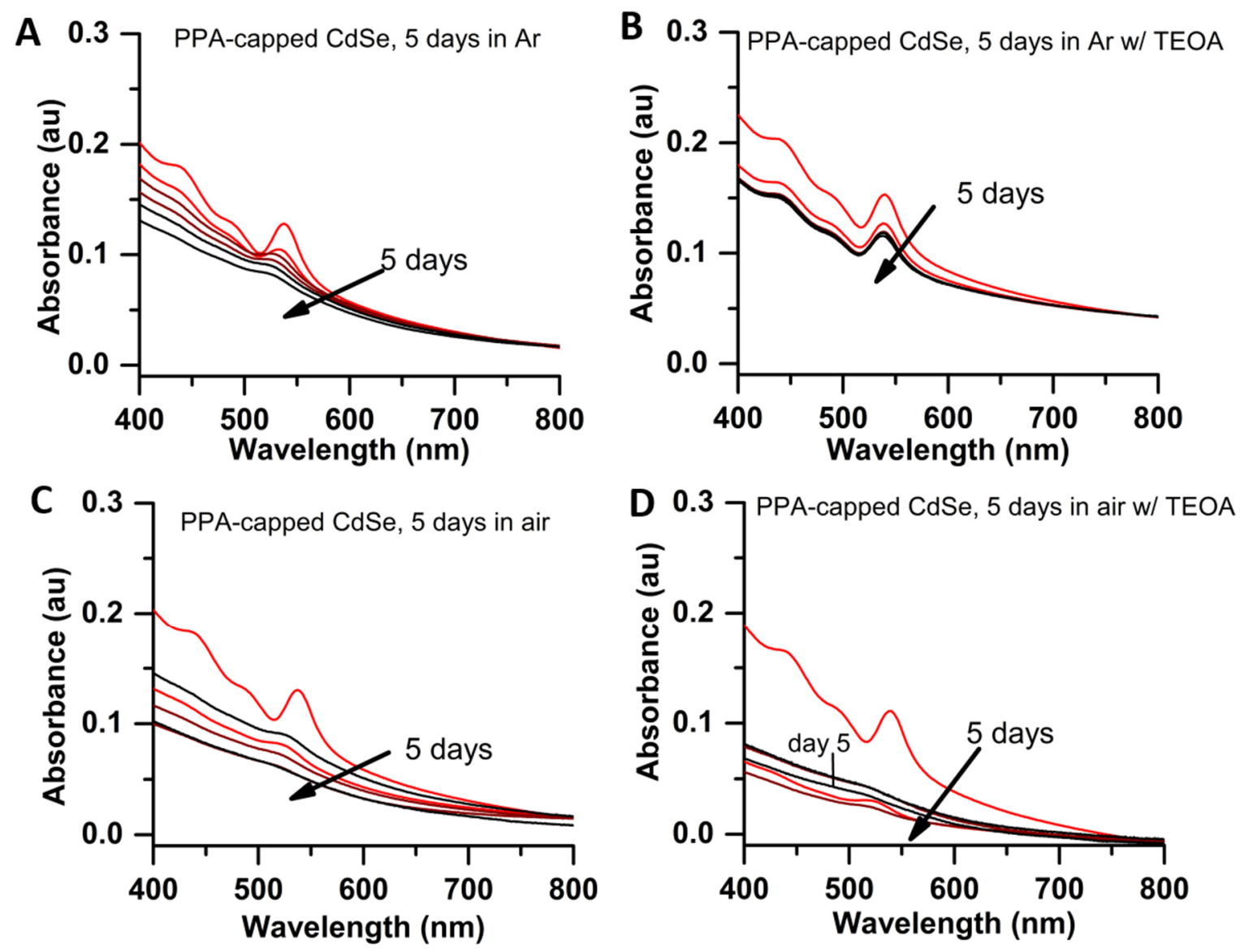

Figure S7. Absorbance of PPA-capped CdSe QDs (aq) over 5 days, when stored in room light in $\operatorname{Ar}(\mathbf{A})$ and air $(\mathbf{C})$, and under the same conditions with added TEOA as hole scavenger (B, Ar; D, air). 
A

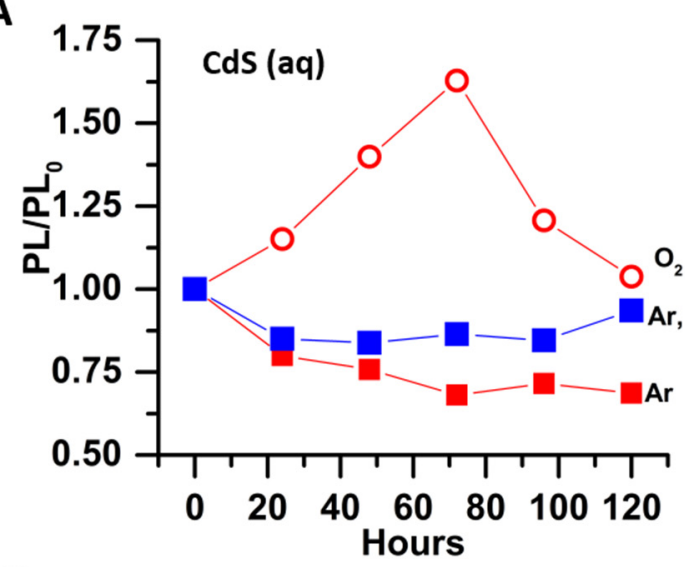

C

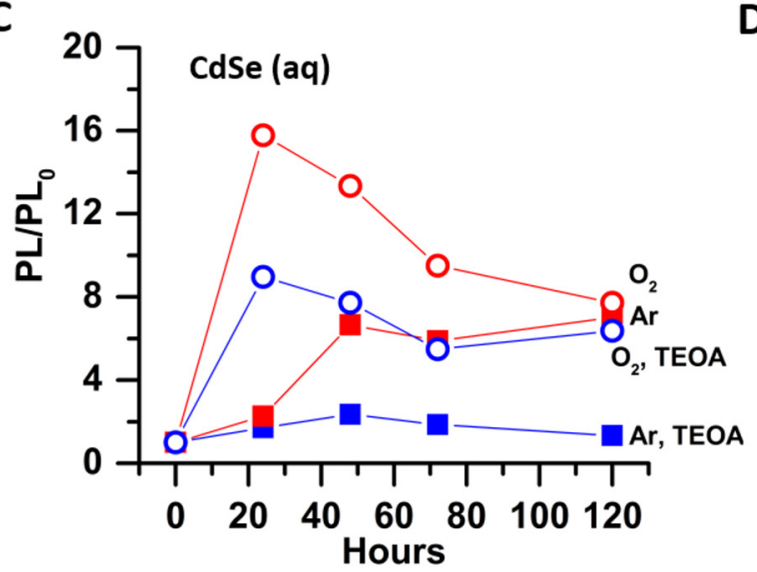

B

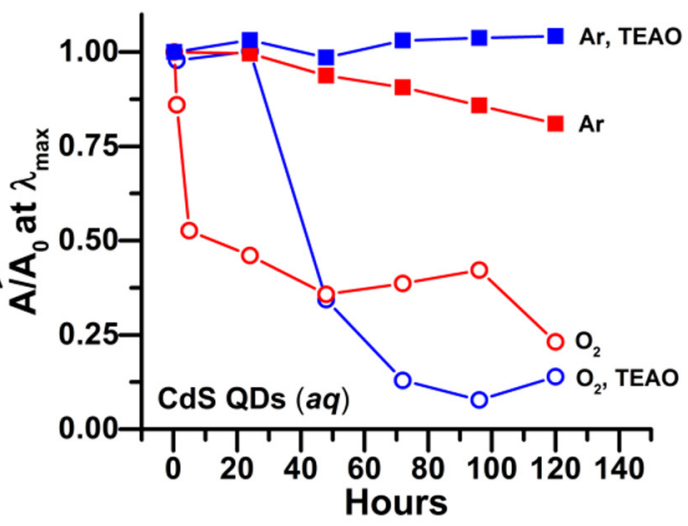

D

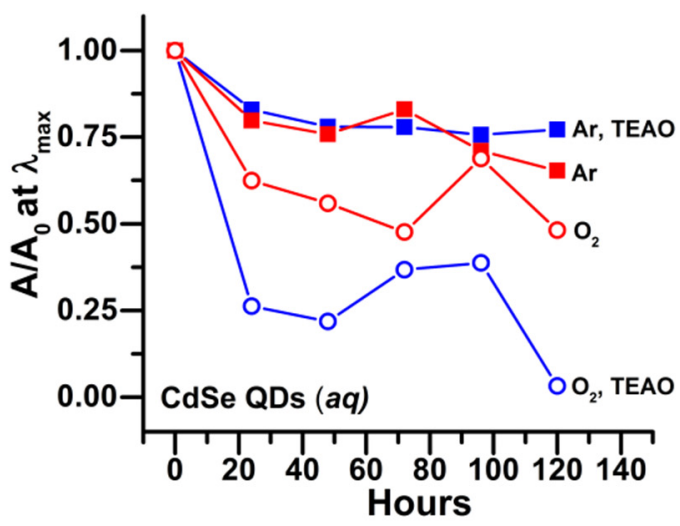

Figure S8. Photoluminescence of CdS QDs (A) and CdSe QDs (C) over 5 days divided by the starting PL intensity, and the corresponding change in absorbance at the first exciton peak over the same period(B CdS, D CdSe). The absorbance figure is reproduced from Figure 4 in the main text. The samples in this figure are the same as those in Figures S4, S5, and Figure 4 in the main text. The PL of the sample of CdS in air with TEOA could not be integrated due to a large background produced by oxidized products of TEOA. Generally, samples stored in room light and air photobrightened and etched. Storing under Ar slowed down both the photobrightening and etching and the addition of TEOA as stabilizer completely stopped the etching and reduced the photobrightening of the QDs. 

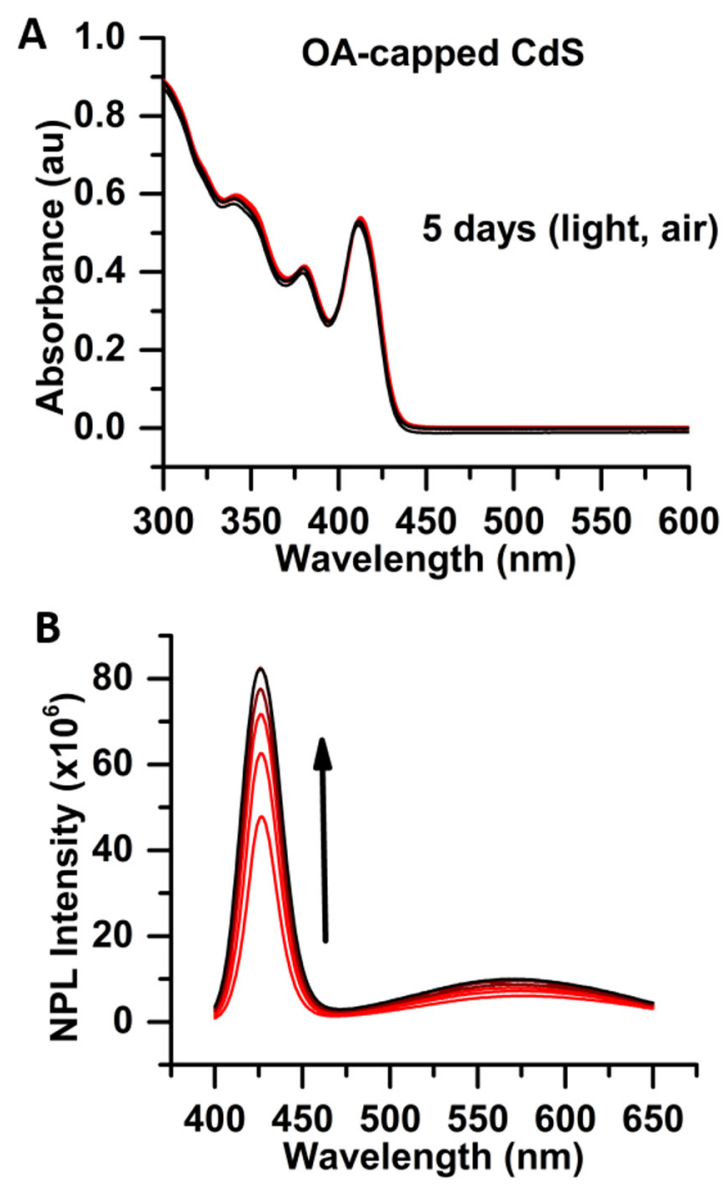

Figure S9. Absorbance over 5 days for oleate-capped CdS QDs stored in air and in room light (A) and corresponding PL spectra of the same samples (B) over the same period. The QDs photobrightened over the 5-day period but did not etch or aggregate.

Fits of Absorbance Spectra to Obtain Absorbance Intensities and Linewidths. We fitted the optical transitions in the absorbance spectra to a set of Gaussian peaks (Figures S10-S17) using the nonlinear curve fit function in Origin. The first exciton peak is in red in all of the figures. We fit the background to a spline polynomial and subtracted this function from each plot to obtain the baselined peaks Figures S10-S17. The raw spectra can be found in Figures S6 and S7. Values of the peak center, FWHM, peak area, and baseline can be found in the tables within the plots. 

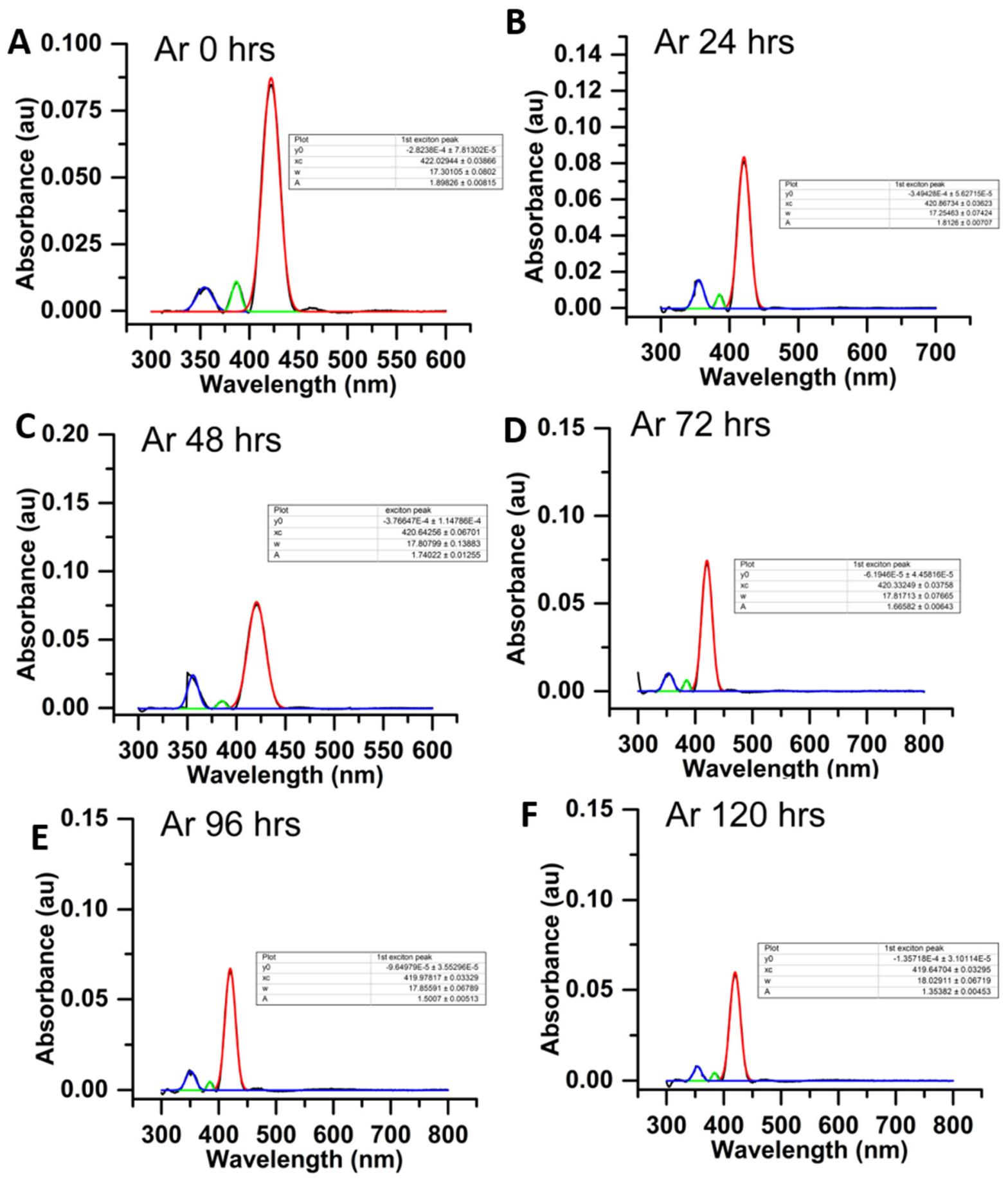

Figure S10. Gaussian fits for PPA-capped CdS QDs in Ar over 5 days. "w" is the FWHM value plotted in Figure 4 in the main text. 

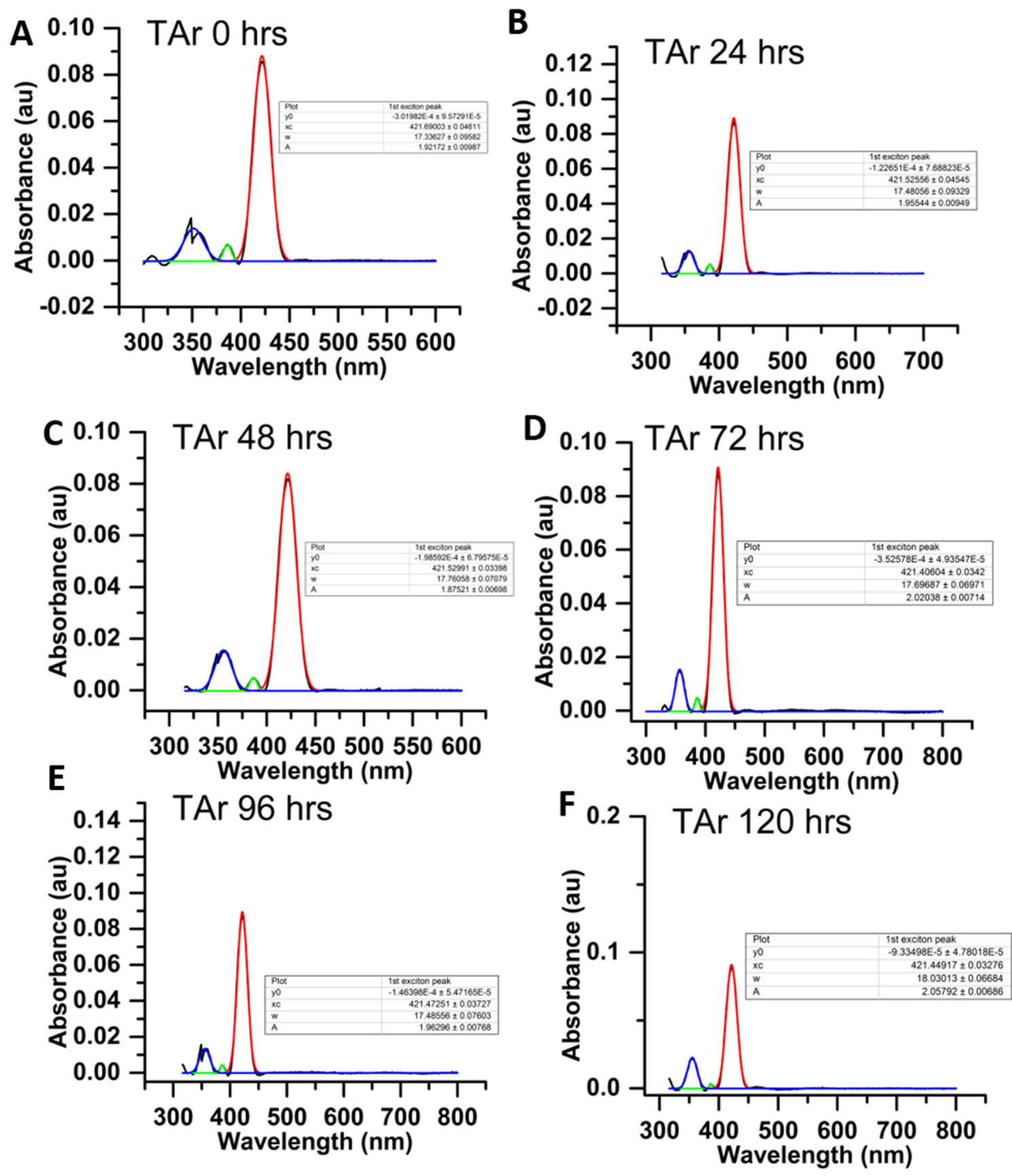

Figure S11. Gaussian fits for PPA-capped CdS QDs in Ar over 5 days with TEOA. "w" is the FWHM value plotted in Figure 4 in the main text. 

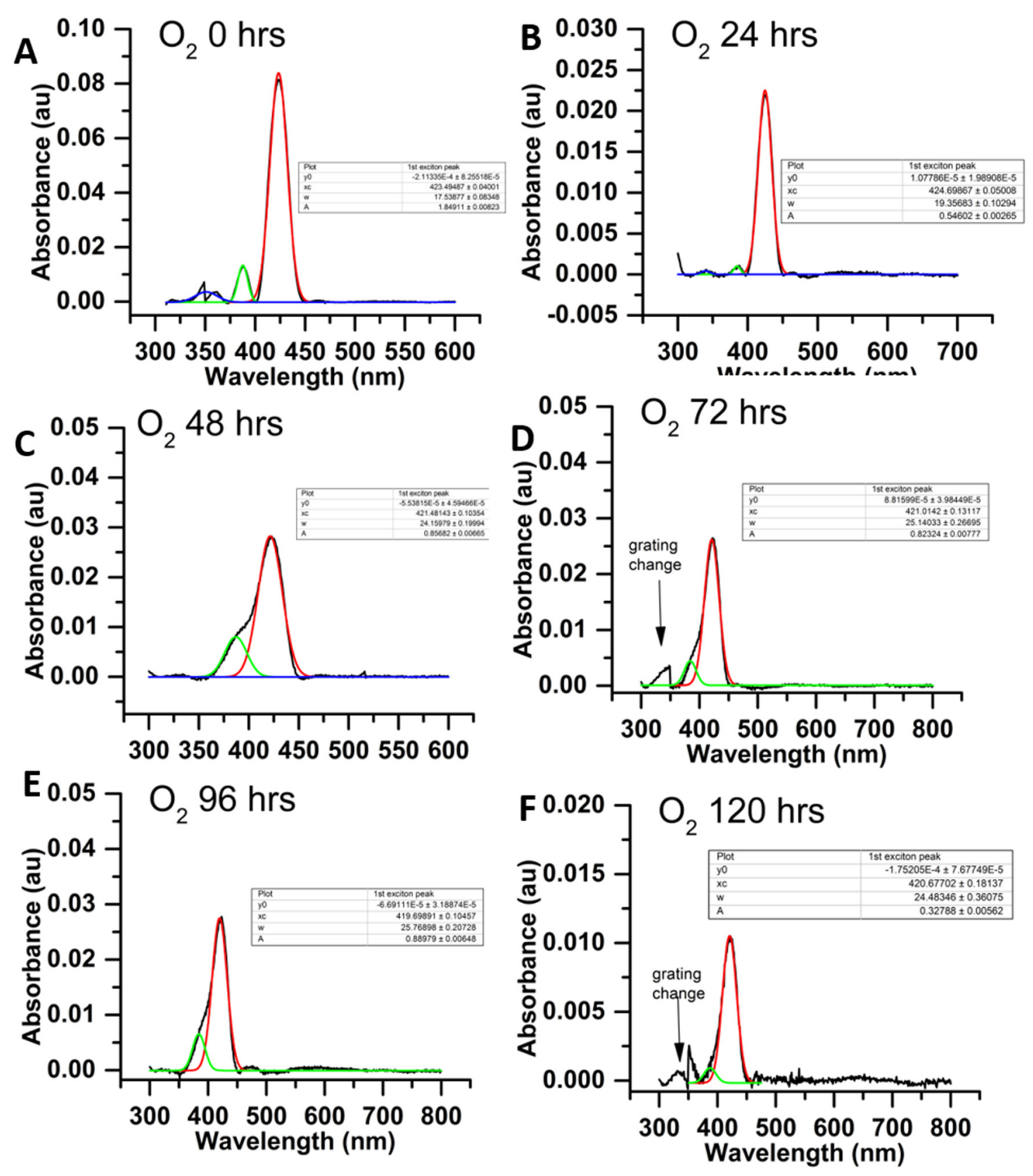

Figure S12. Gaussian fits for PPA-capped CdS QDs in air over 5 days. "w" is the FWHM value plotted in Figure 4 in the main text. 

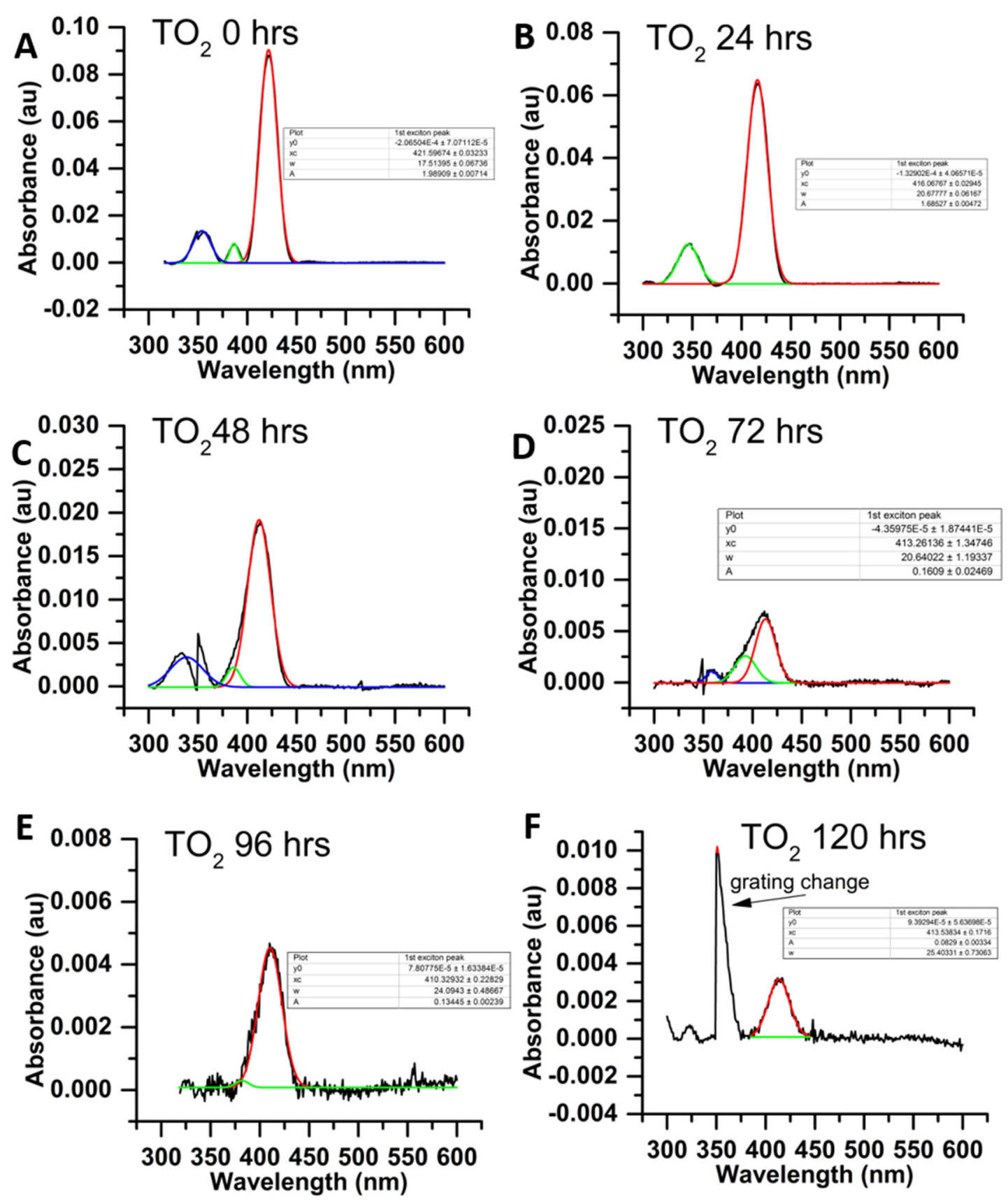

Figure S13. Gaussian fits for PPA-capped CdS QDs in air over 5 days with TEOA. "w" is the FWHM value plotted in Figure 4 in the main text. The sharp feature at $\sim 350 \mathrm{~nm}$ is due to a grating change in the spectrometer. 

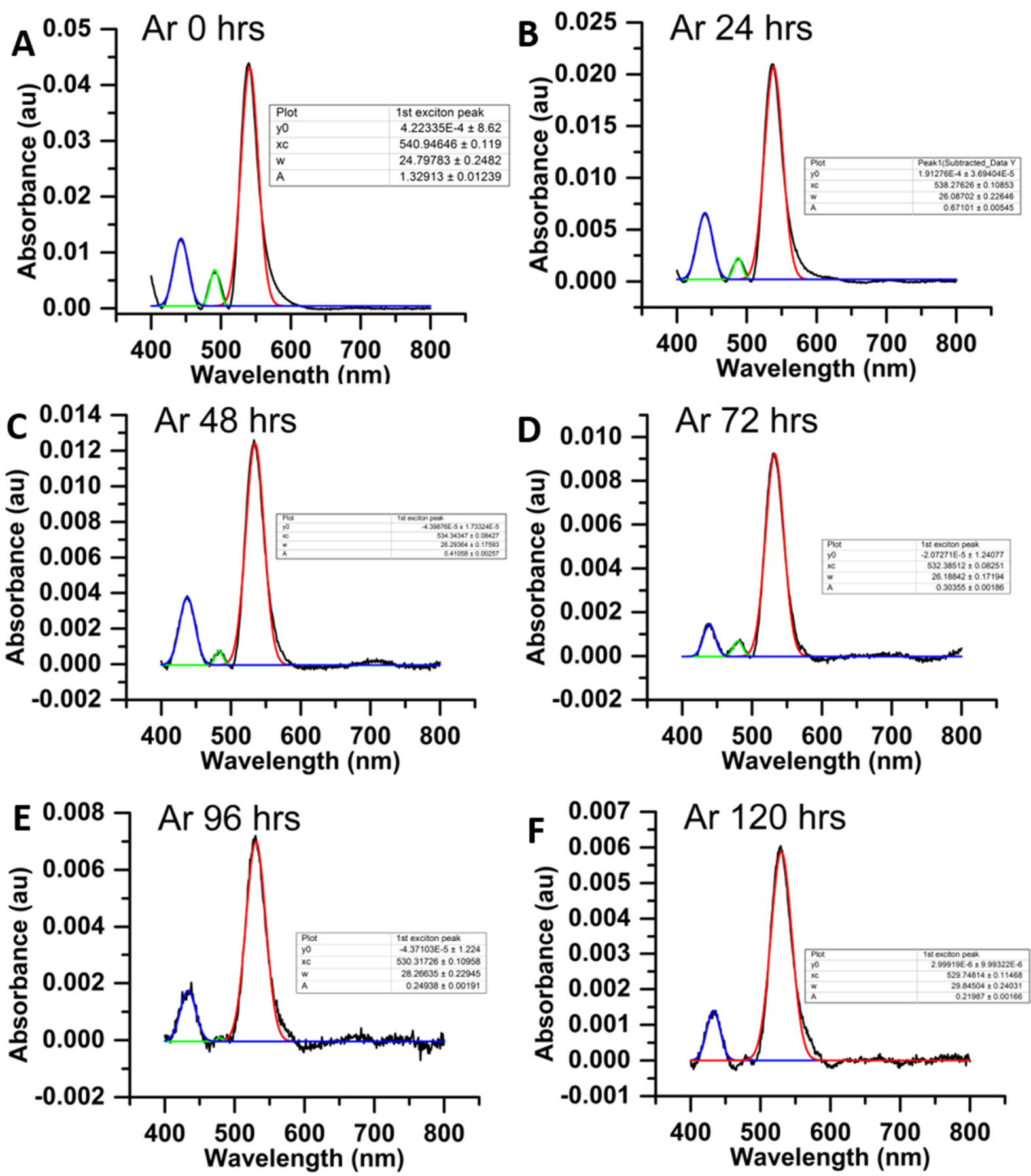

Figure S14. Gaussian fits for PPA-capped CdSe QDs in Ar over 5 days. "w" is the FWHM value plotted in Figure 4 in the main text. 

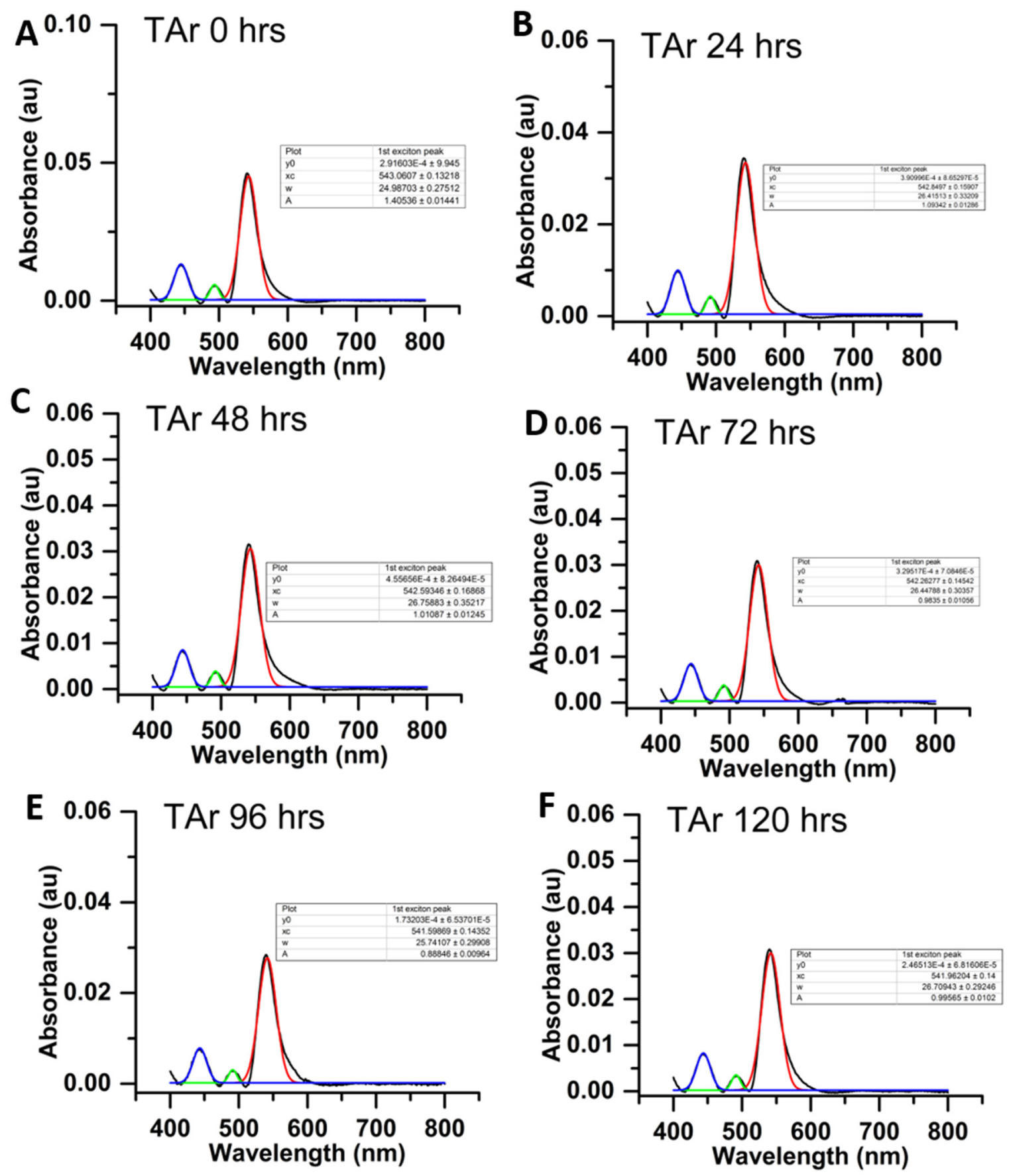

Figure S15. Gaussian fits for PPA-capped CdSe QDs in Ar over 5 days with TEOA. "w" is the FWHM value plotted in Figure 4 in the main text. 

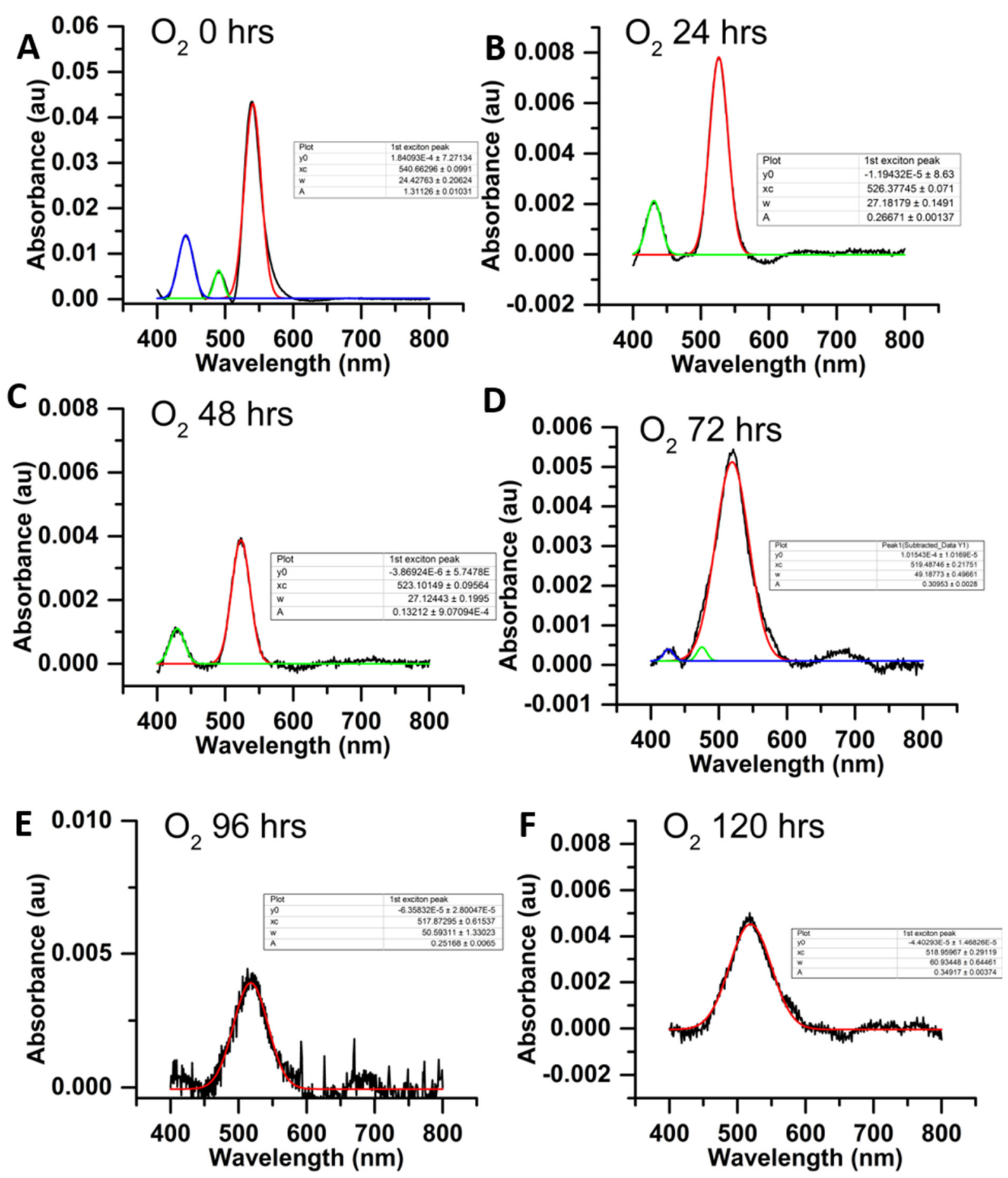

Figure S16. Gaussian fits for PPA-capped CdSe QDs in air over 5 days. "w" is the FWHM value plotted in Figure 4 in the main text. 

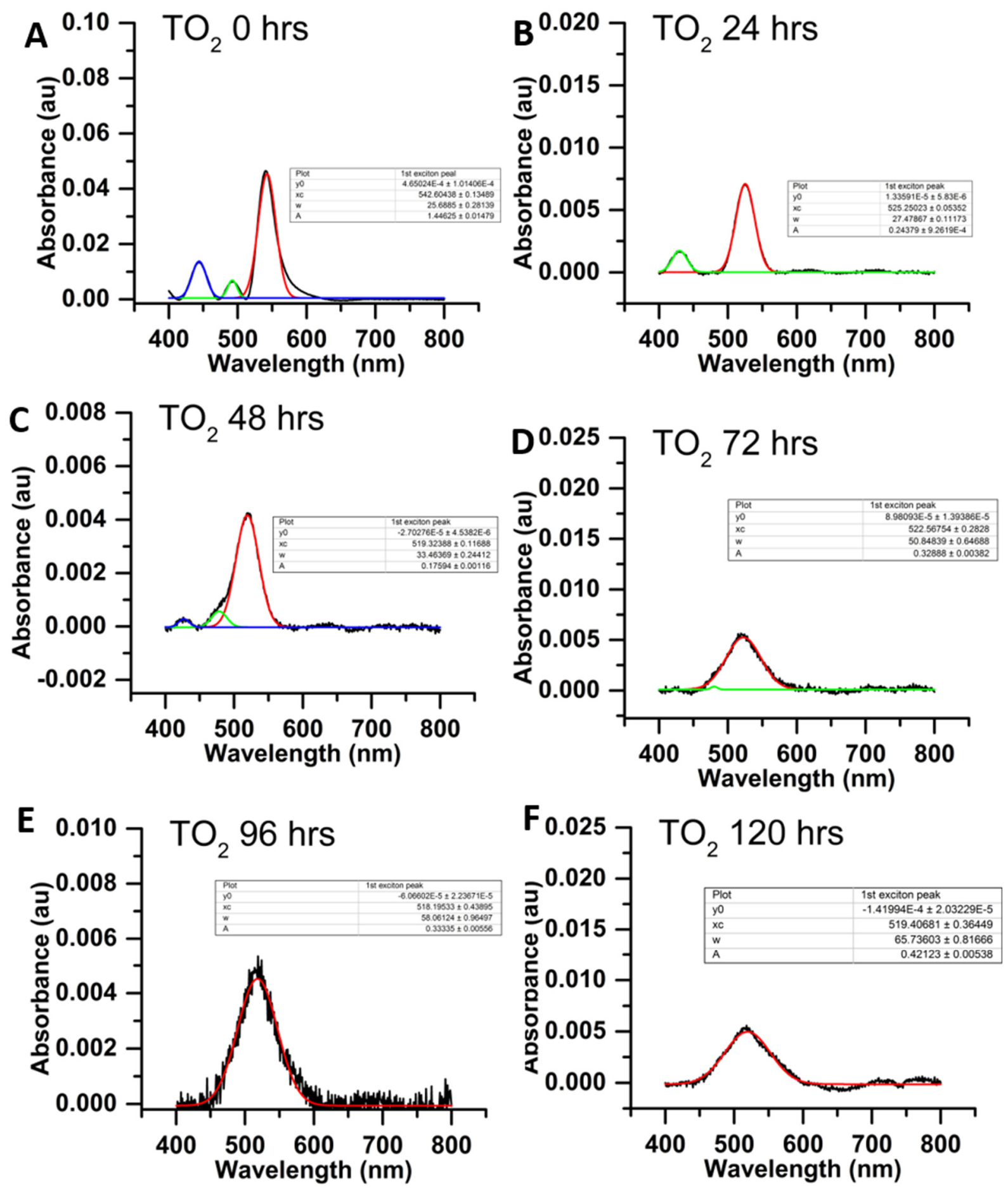

Figure S17. Gaussian fits for PPA-capped CdS QDs in air over 5 days with TEOA. "w" is the FWHM value plotted in Figure 4 in the main text. 
Analysis of NMR Spectra of PPA-capped CdS QDs in Water. We prepared water soluble, PPA-capped QDs at $\mathrm{pH}$ 7. At $\mathrm{pH} 7$, the ${ }^{31} \mathrm{P}$ chemical shift for PPA is at $20.9 \mathrm{ppm} v s$. trimethylphosphate (TMP), a standard added to each sample. We then removed some unknown amount of the excess PPA in the sample by adding activated alumina. After addition of the sorbent, we only observe one peak in the ${ }^{31} \mathrm{P}$ spectrum of the PPA-coated QDs, with a chemical shift of $\sim 19.5 \mathrm{ppm}$. We then split this sample into several sampels, and added a series of equivalents of excess free PPA back into these samples, being careful to keep the $\mathrm{pH}$ the same in all samples. As more free PPA is added, the ${ }^{31} \mathrm{P}$ chemical shift for PPA moves back toward that of free PPA, Figure S18. This dependence of chemical shift on the ratio of bound:free PPA in the sample is also present in the ${ }^{1} \mathrm{H}$ spectrum of the protons alpha to the phosphonate group, Figure S18. This shifting of a single peak in both types of NMR spectra as a function of the concentration of excess PPA is evidence that bound PPA is in fast (relative to the NMR timescale) equlibrium with free PPA in water.

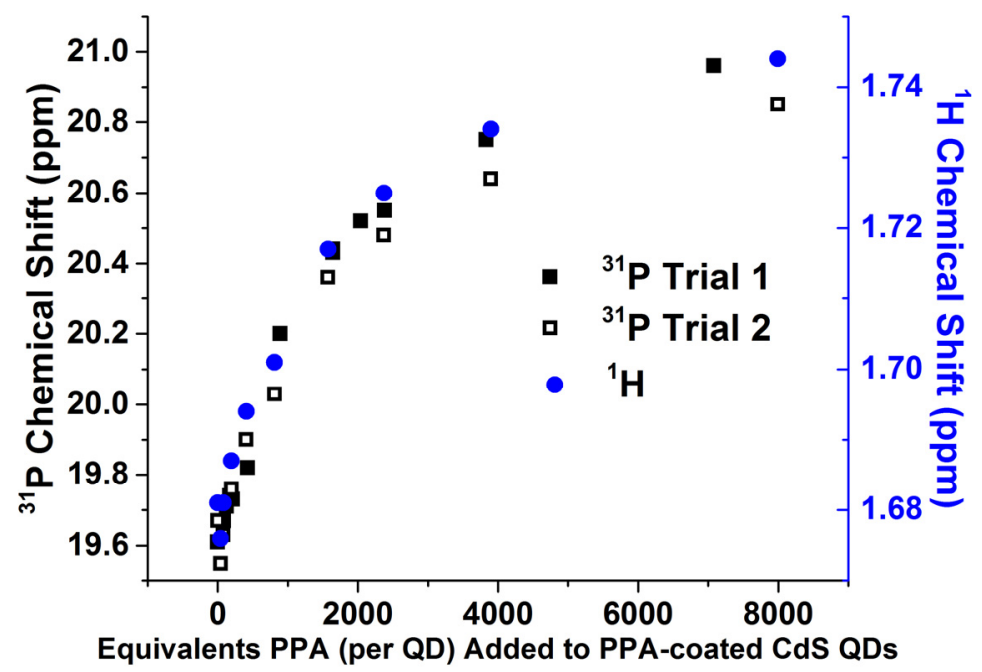

Figure S18. ${ }^{31} \mathrm{P}$ and ${ }^{1} \mathrm{H}$ chemical shifts corresponding to the phosphorous and the protons alpha to the phosphonate group, respectively, of PPA within aqueous dispersions of PPA-capped CdS QDs, as function of the amount of excess PPA in the sample. The " 0 " point (with a ${ }^{31} \mathrm{P}$ chemical shift of $\sim 19.5 \mathrm{ppm}$ ) is obtained by treating the dispersion with activated alumina, which adsorbs excess PPA, until the chemical shift saturates, and is therefore an estimate of the chemical shift of purely bound PPA. 


\section{References}

1. Harris, R. D.; Amin, V. A.; Lau, B.; Weiss, E. A., Role of Interligand Coupling in Determining the Interfacial Electronic Structure of Colloidal CdS Quantum Dots. Acs Nano 2016, 10, 1395-1403.

2. Chen, O.; Chen, X.; Yang, Y.; Lynch, J.; Wu, H.; Zhuang, J.; Cao, Y. C., Synthesis of Metal-Selenide Nanocrystals Using Selenium Dioxide as the Selenium Precursor. Angew Chem Int Ed 2008, 47, 8638-8641.

3. Yu, W. W.; Qu, L. H.; Guo, W. Z.; Peng, X. G., Experimental Determination of the Extinction Coefficient of CdTe, CdSe, and CdS Nanocrystals. Chem Mater 2003, 15, 2854-2860.

4. Melhuish, W. H., Quantum Efficiencies of Fluorescence of Organic Substances - Effect of Solvent and Concentration of Fluorescent Solute. J. Phys. Chem. 1961, 65 (2), 229-230.

5. Aruda, K. O.; Amin, V. A.; Thompson, C. M.; Lau, B.; Nepomnyashchii, A. B.; Weiss, E. A., Description of the Adsorption and Exciton Delocalizing Properties of $p$-Substituted Thiophenols on CdSe Quantum Dots. Langmuir 2016, 32, 3354-3364.

6. Vairavamurthy, M. A.; Goldenberg, W. S.; Ouyang, S.; Khalid, S., The Interaction of Hydrophilic Thiols with Cadmium: Investigation with a Simple Model, 3-Mercaptopropionic Acid. Mar Chem 2000, 70, 181-189. 